THE MAXIMAL GENERIC NUMBER

OF PURE NASH EQUILIBRIA

by

Andrew McLennan

Discussion Paper No. 273, July 1994

Center for Economic Research Department of Economics University of Minnesota Minneapolis, MN 55455 


\title{
The Maximal Generic Number of Pure Nash Equilibria*
}

\author{
by \\ Andrew McLennan \\ Department of Economics \\ University of Minnesota \\ 271 19th Avenue South \\ Minneapolis, MN 55455 \\ mclennancatlas. socsci.umn. edu
}

July 5, 1994

\begin{abstract}
For finite pure strategy sets $S_{1}, \ldots, S_{n}$, if $E \subset S=S_{1} \times \ldots \times S_{n}$ is the set of pure strategy Nash equilibria for an open set of payoffs vectors, then $\# E \leq \# S /\left(\max _{i} \# S_{i}\right)$. There is an open set of payoff vectors for which there are $\# S /\left(\max _{i} \# S_{i}\right)$ pure Nash equilibria.
\end{abstract}

* This research was supported in part by National Science Foundation grant SBR-9308862 to the University of Minnesota. I have benefited from numerous discussions with Richard McKelvey. 


\title{
The Maximal Generic Number of Pure Nash Equilibria
}

by

\author{
Andrew McLennan
}

A normal form game can have as many pure strategy Nash equilibria as there are pure strategy vectors, if each agent's payoff never depends on her own choice of strategy, but this phenomenon is not robust with respect to perturbations of payoffs. This note characterizes the largest sets of pure strategy vectors that are the sets of pure strategy equilibria for open sets of payoffs. In addition to resolving a point of curiosity, this and related results have implications for the worst case running times of algorithms that compute all Nash equilibria.

Several recent papers deal with the number of Nash equilibria possessed by normal form games. Fixing strategy spaces, McKelvey and McLennan (1994) characterize the maximal (as the payoffs are varied) number of regular totally mixed equilibria. It is a longstanding open problem to show that for two agents, each with $k$ pure strategies, there is an open dense subset of payoffs on which there are at most $2^{k}-1$ Nash equilibria. Quinn and Shubik confirm ${ }^{1}$ this conjecture for the case $k=3$. Extending earlier results, Stanford (1993) characterizes the asymptotic (as the strategy spaces of at least two agents increase in size) probability that a "randomly selected" payoff vector will have exactly $m$ pure strategy equilibria. Stanford (1994) extends this analysis to symmetric two person games, differentiating between symmetric and asymmetric equilibria.

Let the set of agents be $I=\{1, \ldots, n\}$. Let finite strategy sets $S_{1}, \ldots, S_{n}$ be given, and let $S=S_{1} \times \ldots \times S_{n}$. We say that $E \subset S$ is thin if, for any distinct $s, t \in E$, there are at least two $i$ such that $s_{i} \neq t_{i}$. That is, there do not exist distinct $s, t \in E$ with $s_{j}=t_{j}$ for all $j$ except some particular agent $i$.

Lemma: If $u_{1}, \ldots, u_{n} \in \mathbb{R}^{S}$ are payoffs such that $u_{i}(s) \neq u_{i}(t)$ for all $i$ and all distinct $s, t \in S$, and $E$ is the set of pure Nash equilibria for $u_{1}, \ldots, u_{n}$, then $E$ is thin.

Proof: Otherwise there exist $s, t \in E$ and an agent $i$ such that $s_{i} \neq t_{i}$ while $\left(s_{j}\right)_{j \neq i}=$ $\left(t_{j}\right)_{j \neq i}$. The hypothesis does not allow both $s_{i}$ and $t_{i}$ to be best responses to $\left(s_{j}\right)_{j \neq i}$.

1 Personal communication. 
Proposition: If $E$ is nonempty and thin, then there is an open set of $\left(u_{1}, \ldots, u_{n}\right) \in\left(\mathbb{R}^{S}\right)^{I}$ for which $E$ is the set of pure Nash equilibria.

Proof: Let $E_{0}=E$, and define $E_{1}, \ldots, E_{n}$ inductively by

$$
\begin{gathered}
E_{k}=\left\{s \in S-\left(E_{0} \cup \ldots \cup E_{k-1}\right): \text { there is } t \in\left(E_{0} \cup \ldots \cup E_{k-1}\right)\right. \text { such that } \\
\left.s_{i} \neq t_{i} \text { for exactly one } i\right\} .
\end{gathered}
$$

Evidently $E_{0}, \ldots, E_{n}$ is a partition of $S$. Define $\left(u_{1}, \ldots, u_{n}\right)$ by setting $u_{i}(s)=-k$ for all $i$ and $s \in E_{k}$. Then it is easily seen that $E$ will be the set of pure Nash equilibria for all vectors of payoffs in a neighborhood of $\left(u_{1}, \ldots, u_{n}\right)$.

We say that a thin set is maximal if there is no thin proper superset. We say that a thin set is svelte if there is no thin set with a larger number of elements. It seems reasonable to conjecture that maximal thin sets are svelte, but I do not know if this is the case.

Our main result is:

Theorem: Assume that $\# S_{1} \geq \ldots \geq \# S_{n}$. Then:

A. Svelte subsets of $S$ have $\# S_{2} \times \ldots \times \# S_{n}$ elements;

B. There are $\# S_{n}$ pairwise disjoint svelte sets.

Proof: We argue by induction on $n$. Both claims are trivial when $n=1$, so assume they have been established for the case of $n-1$ agents. Let $S_{n}=\left\{s_{n}^{1}, \ldots, s_{n}^{m}\right\}$. If $E$ is svelte, then $E=\cup_{h=1}^{m} F_{h} \times\left\{s_{n}^{h}\right\}$, where $F_{1}, \ldots, F_{m}$ are pairwise disjoint thin subsets of $S_{1} \times \ldots \times S_{n-1}$, so svelte subsets of $S$ have at most $\left(\# S_{2} \times \ldots \times \# S_{n-1}\right) \times \# S_{n}$ elements. If, on the other hand, $F_{1}, \ldots, F_{m}$ are pairwise disjoint thin subsets of $S_{1} \times \ldots \times S_{n-1}$, then for each $q=1, \ldots m$,

$$
E_{q}=\left(F_{1} \times\left\{s_{n}^{q+1}\right\}\right) \times \ldots \times\left(F_{m-q} \times\left\{s_{n}^{m}\right\}\right) \times\left(F_{m-q+1} \times\left\{s_{n}^{1}\right\}\right) \times \ldots \times\left(F_{m} \times\left\{s_{n}^{q}\right\}\right)
$$

is thin, and $E_{1}, \ldots, E_{m}$ are pairwise disjoint. 


\section{References}

McKelvey, R.D., and A. McLennan, (1994), "The maximal number of regular totally mixed Nash equilibria," Social Science Working Paper \#865, California Institute of Technology.

Stanford, W., (1993), "The Poisson distribution and probability of $k$ pure equilibria in matrix games," mimeo, Department of Economics, University of Illinois at Chicago.

Stanford, W., (1994), "The limit distribution of the number of pure equlibria in symmetric bimatrix games," mimeo, Department of Economics, University of Illinois at Chicago. 